\title{
Abducens nerve palsy as initial symptom of trigeminal schwannoma
}

\author{
J U N K O Y A M A S I TA, RENIN ASATO, HAJIME HANDA, \\ SATOSHI NAKAO, A N MASAHIRO OGATA \\ From the Departments of Neurosurgery, Kyoto University Medical School, Kyoto, and Kobe \\ Municipal Central Hospital, Kobe, Japan
}

SUMMARY The insidious onset and slow progression of symptoms may cause delay in the recognition of trigeminal schwannomas. Two cases with abducens nerve palsy as an initial symptom are reported, in both of whom the tumour was growing mainly below and anterior to the intracavernous portion of the internal carotid artery. Early detection of clinical symptoms and thorough radiological investigations are important in the early diagnosis of these lesions.

Schwannomas of the trigeminal nerve are rare tumours. They usually present pains or sensory loss of the face as initial symptoms. Cases without trigeminal involvement have been reported occasionally (Jefferson, 1955; Olive and Svien, 1957; Knudsen and Kolze, 1972; Arseni et al., 1975). Disturbances of extraocular movements are frequently observed as a minor concomitant symptom in the advanced stage of trigeminal schwannomas, as the result of cavernous sinus compression. However, this is rarely seen as an initial symptom. Recent experience of two such unusual cases with abducens nerve palsy as an initial and predominant symptom prompted us to report them and to elucidate its diagnostic and therapeutic importance.

\section{Case reports}

CASE 1

This 38 year old man was admitted to Kyoto University Hospital on 12 March 1976, complaining of double vision which he had first noted on right lateral gaze while driving about 10 months previously. The symptom was gradually progressive, and he consulted a hospital where it was pointed out that he had right abducens nerve palsy. $\mathrm{He}$ was referred to our hospital with the clinical diagnosis of parasellar tumour. His past medical

Address for reprint requests: Professor H. Handa, Department of Neurosurgery, Kyoto University Medical School, 54 Kawahara-cho, Sakyoku, Kyoto, Japan 606.

Accepted 12 July 1977 history and family history were non-contributory.

Examination on admission was within normal limits except for right abducens nerve palsy. Sensation of the face and corneal reflex were intact. There were no cerebellar signs or signs of increased intracranial pressure. Plain skull radiographs showed no deformity of the sella turcica. There was no bony destruction in the middle cranial fossa and pyramis. Right carotid angiography (Fig. 1) showed the intracavernous portion of the internal carotid artery displaced medially, superiorly and posteriorly. There was a tumour stain of fingertip size in the parasellar region in the late arterial and capillary phases of the lateral projection. Retrograde jugular venography disclosed a shadow defect in the anterolateral portion of the right cavernous sinus. Pneumoencephalography showed a slight dilatation and elevation of the right temporal horn of the lateral ventricle.

On 24 March 1976, a right temporal craniotomy was carried out in the left lateral position. When the temporal lobe was elevated through the intradural approach, an extradural mass was seen in the medial portion of the middle cranial fossa. The tumour was well demarcated and vascular. It was incompletely removed, leaving a small portion adhering to the medial structures. Nerve fibres in the extradural space under the tumour were thought to be the first division of the trigeminal nerve. His postoperative course was uneventful but an area of hypaesthesia persisted in the region supplied by the first and second 

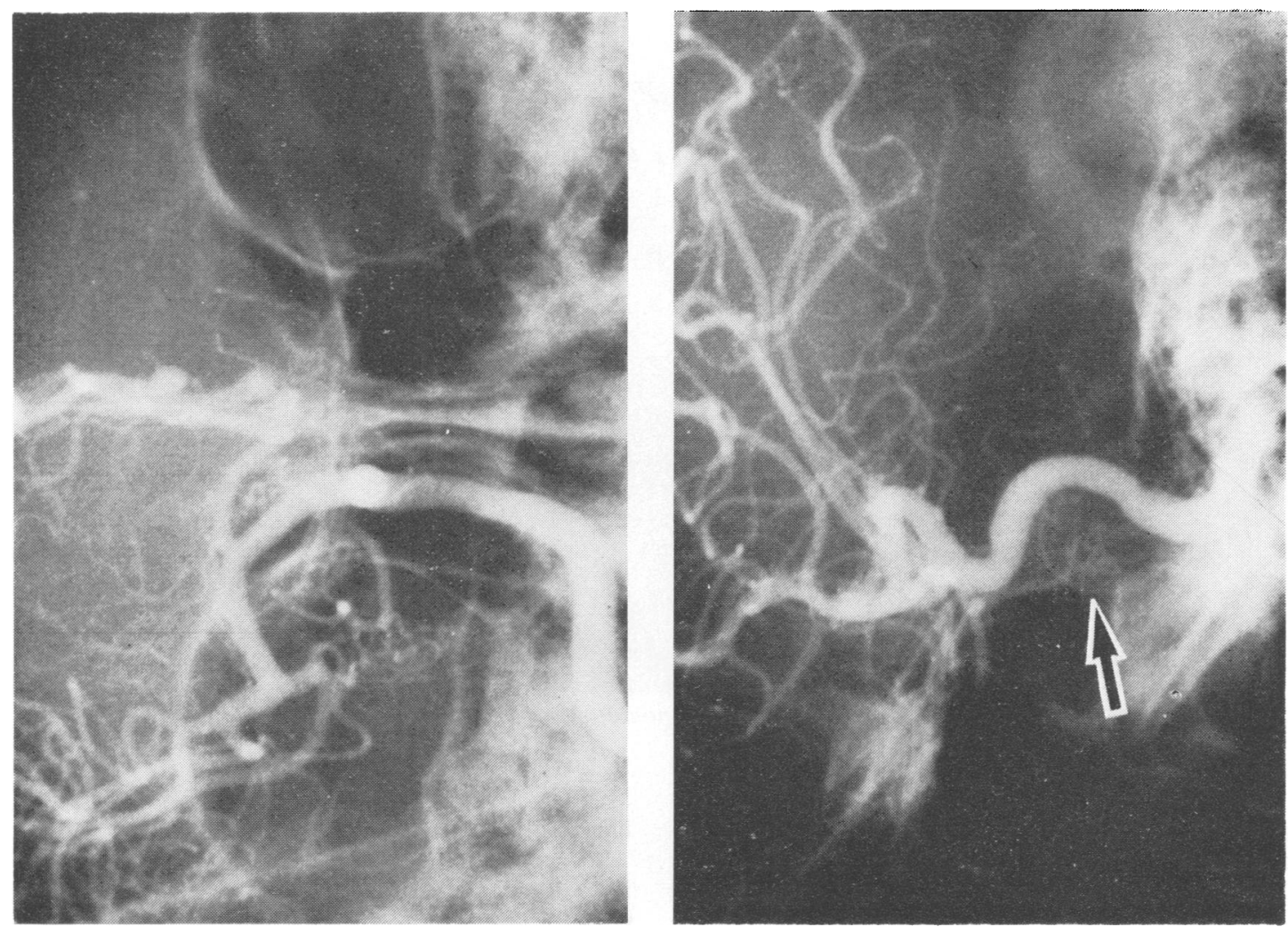

Fig. 1 Case 1. Right carotid angiography showing intracavernous portion of internal carotid artery displaced medially, superiorly and posteriorly. There is a tumour stain in the parasellar region (arrow).

branches of the trigeminal nerve and the right corneal reflex was absent. Abducens nerve palsy was much improved postoperatively. Histological diagnosis was reported to be a schwannoma (Fig. 2). When he was seen in the outpatient clinic eight months later, right abducens nerve palsy had disappeared completely, although the disturbance of facial sensation and corneal reflex persisted. $\mathrm{He}$ had returned to his previous occupation and was able to drive a car.

\section{CASE 2}

This 27 year old man was admitted to Kobe Municipal Central Hospital on 9 October 1974, complaining of double vision. He was well until five days before admission when headache, nausea, and vomiting developed acutely without loss of consciousness while playing mah-jong. The next day he woke up with double vision on left lateral gaze. Symptoms other than diplopia had gradually subsided in a few days. His past medical and family histories were non-contributory.
Examination on admission showed that he was alert and orientated. General physical and neurological examinations showed no abnormalities except left abducens nerve palsy. Corneal reflex and facial sensation were intact. There were no cerebellar signs or signs of increased intracranial pressure. On the eighth and tenth hospital days he experienced two episodes of acute exacerbation of headache, nausea, and vomiting. On each occasion the degree of left abducens palsy became even worse. Around the 20th hospital day, the patient noted for the first time decreased sensation to pinprick and touch in the region supplied by the first division of the left trigeminal nerve.

The routine laboratory examinations were all within normal limits. Spinal tap performed on the third hospital day showed the initial pressure $110 \mathrm{mmH}_{2} \mathrm{O}$ and the closing pressure $90 \mathrm{mmH}_{2} \mathrm{O}$. Cerebrospinal fluid appeared watery clear: its protein content was $0.33 \mathrm{~g} / 1$ and there was no pleocytosis. Skull radiographs showed a slight enlargement of the left foramen ovale. Left 


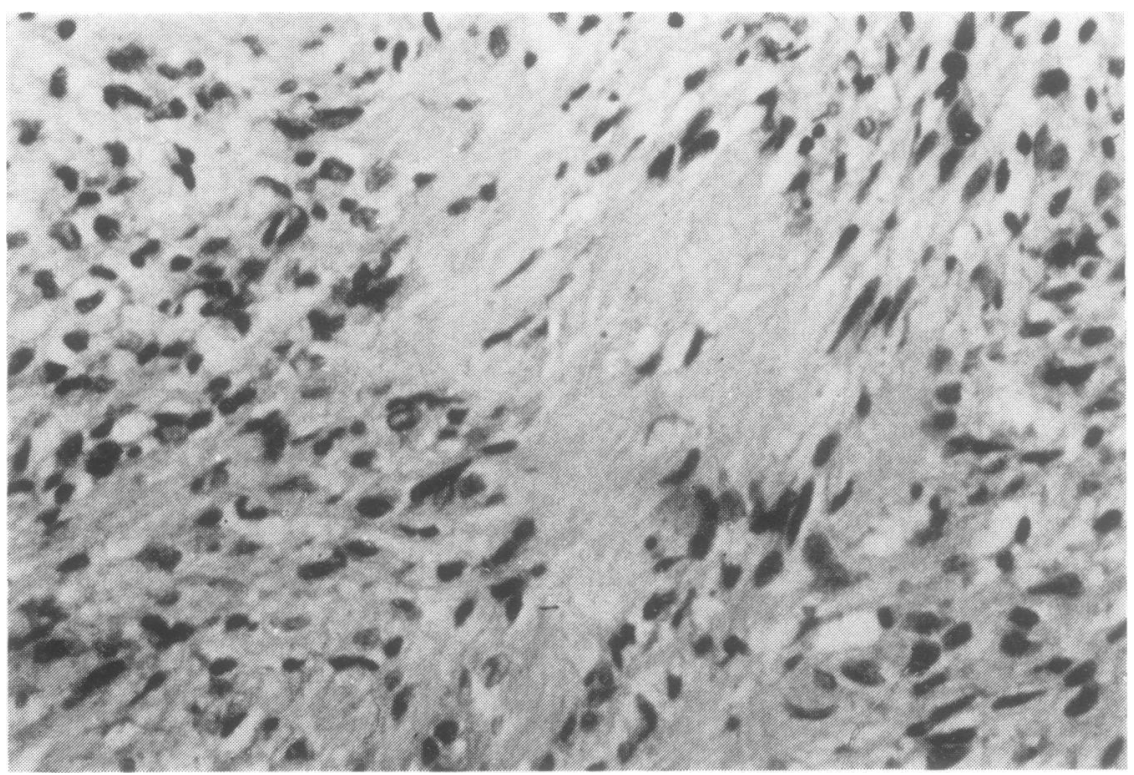

Fig. 2 Photomicrograph showing schwannoma (case 1). $H E \times 200$.

carotid angiography (Fig. 3) showed the intracavernous portion of the internal carotid artery displaced medially, superiorly and posteriorly. There was a tumour stain of fingertip size in the region of the cavernous sinus in the lateral projection. Retrograde jugular venography showed a filling defect in the anterolateral portion of the left cavernous sinus, corresponding to the site of the tumour stain on angiography.

A left temporal craniotomy was carried out on 28 November 1974. The lesion was approached intradurally. On elevating the temporal lobe, an extradural tumour was found at the site of the foramen ovale. The tumour was elastic soft, well demarcated, and vascular. No evidence of recent intratumoural haemorrhage was confirmed during operation. The tumour was incompletely removed, leaving a portion adhering to the first division of the trigeminal nerve.

Postoperatively, left abducens nerve palsy was temporarily worsened, and there was sensory loss in the region supplied by the first and second divisions of the trigeminal nerve. Histological diagnosis was schwannoma (Fig. 4). The patient received a course of postoperative irradiation. When he was seen two months after the completion of irradiation, left abducens nerve palsy had disappeared and the only neurological abnormality was sensory dullness in the region supplied by the first division of the left trigeminal nerve.

\section{Discussion}

The incidence of trigeminal schwannomas reported by various authors ranged between 0.07 and $0.33 \%$ of all intracranial tumours (Schisano and Olivecrona, 1960; Arseni et al., 1975). In the Department of Neurosurgery, Kyoto University Medical School, nine cases of trigeminal schwannomas were recorded among 2491 intracranial tumours in the period of 36 years from 1941 to 1976 inclusive $(0.36 \%)$. Among 10 cases including one from Kobe Municipal Central Hospital reported as case 2 above, five were men and five women. The age varied from 24 to 43 years with an average of 33.4 years. The clinical data of these 10 cases are summarised in the Table.

Jefferson (1955) classified trigeminal schwannomas into three groups: (1) in the middle cranial fossa, (2) in the posterior cranial fossa, and (3) in both middle and posterior cranial fossae. Symptoms vary depending on the anatomical localisation of the tumour. The different clinical manifestations have been analysed extensively in the literature. Since our two cases are of trigeminal schwannomas in the middle cranial fossa, our discussion will focus mainly on this variety.

In more than half the cases reported, facial pain or facial numbness was the initial and predominant clinical symptom. Krayenbühl (1936), in his study of trigeminal schwannomas, emphasised that 


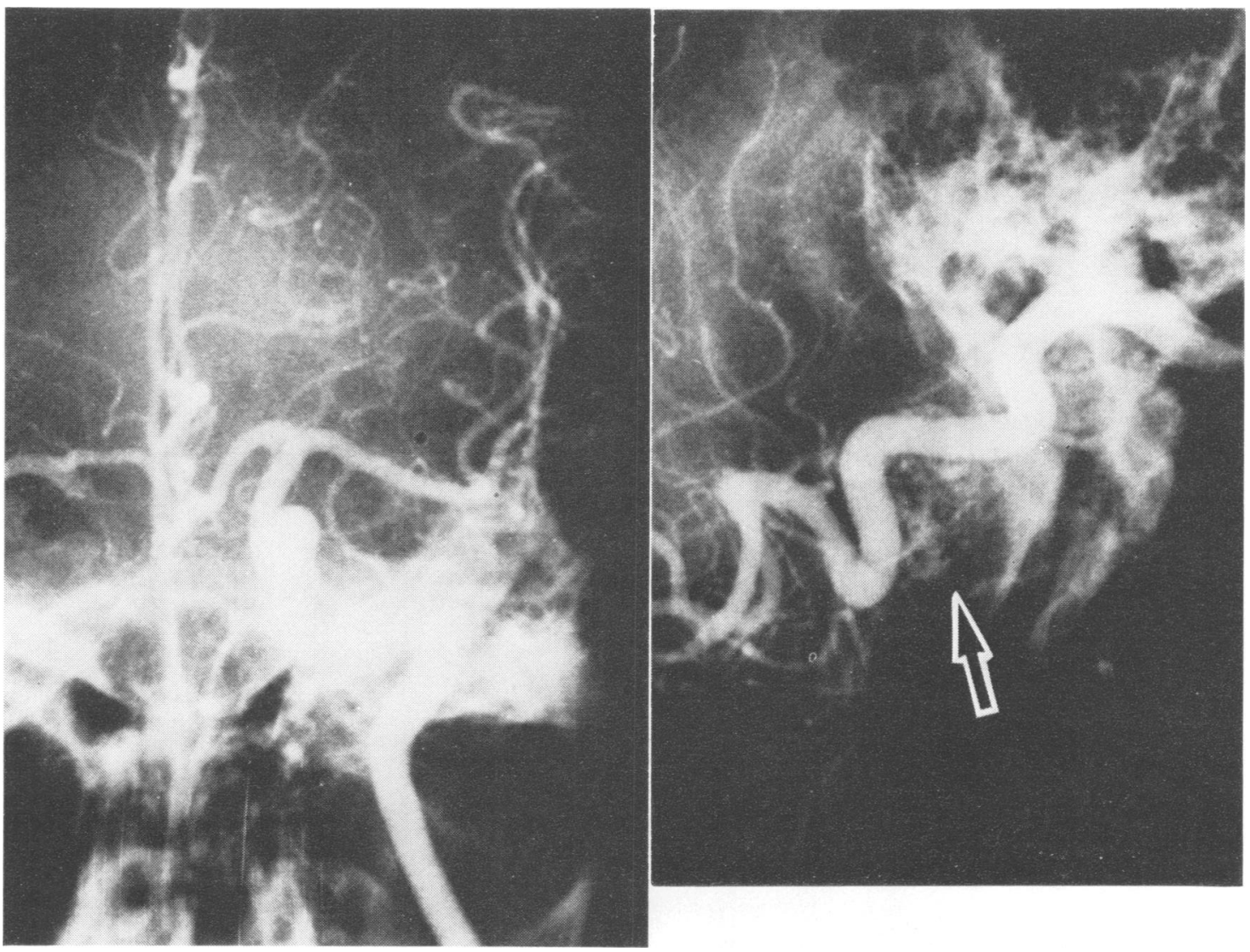

Fig. 3 Case 2. Left carotid angiography showing intracavernous portion of internal carotid artery displaced medially, superiorly and posteriorly. There is a tumour stain in the parasellar region (arrow).

intense facial pain is in favour of a tumour in the middle cranial fossa starting from the Gasserian ganglion, whereas a tumour of the trigeminal root in the posterior cranial fossa is often unaccompanied by pain. This observation was confirmed by Schisano and Olivecrona (1960). None of their four patients with schwannomas of the trigeminal root suffered from pain, whereas five out of 11 cases with schwannomas of the Gasserian ganglion experienced facial pain. In our series, however, only one out of five cases with schwannomas of the Gasserian ganglion suffered from pain, and none of those in the posterior fossa or in both fossae experienced facial pain. In our opinion the presence of facial pain does not appear to be important in tumour localisation. Arseni et al. (1975) also expressed the same opinion regarding facial pain.

Our two cases had abducens nerve palsy as an initial and predominant symptom while trigeminal symptomatology was absent (case 1) or slight (case 2). We could find only three similar cases among 53 cases from the available literaturenamely, case 3 and case 9 of Schisano and Olivecrona's series (1960), and Gordy's case (1965). It is well known that some cases of trigeminal schwannomas have no facial pain or numbness (Jefferson, 1955; Olive and Svien, 1957; Knudsen and Kolze, 1972; Arseni et al., 1975). Jefferson (1955) explains the absence of, or mild, trigeminal symptomatology by the fact that the schwannoma involves only part of the nerve fibres, and the tumour in its subsequent development does not affect the root of the nerve.

The oculomotor nerve is rarely affected even if the cavernous sinus is compressed by these tumours. According to Jefferson (1955), this is in strong contrast with malignant invasions of the 


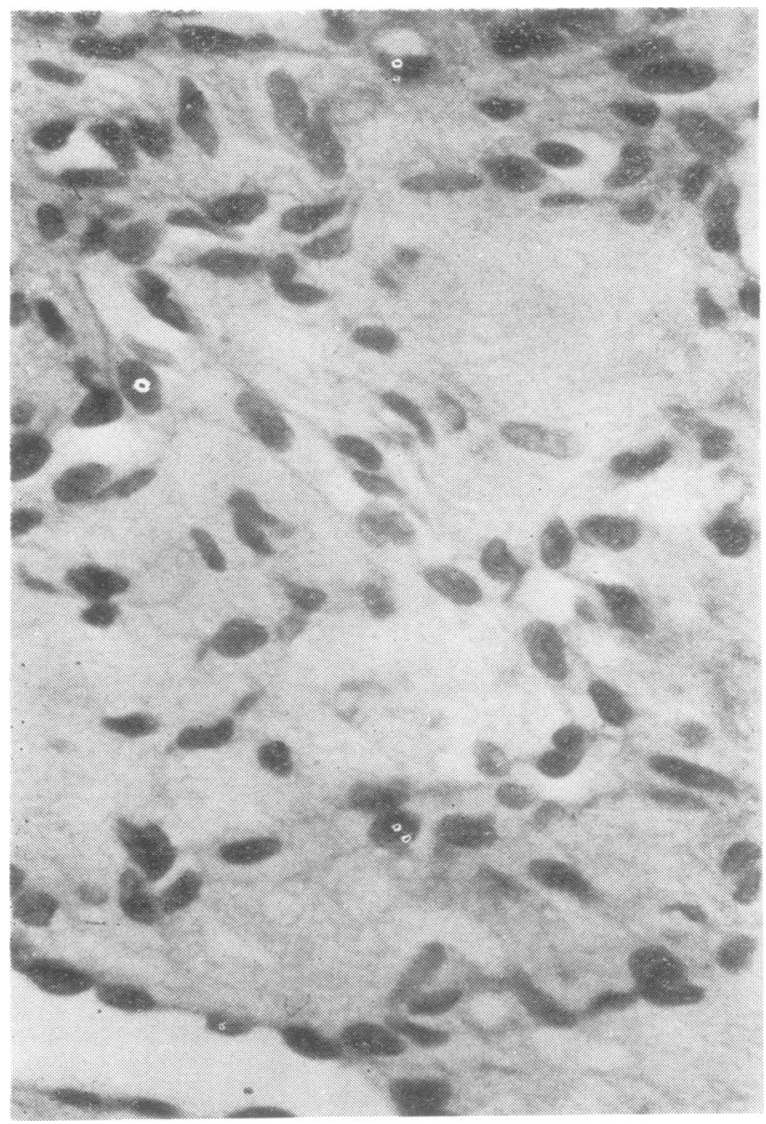

Fig. 4 Photomicrograph showing schwannoma (case 2). $H E \times 400$.

ganglion or secondary deposits which frequently produce total ophthalmoplegia. Among the cranial nerves controlling the extraocular movements, all of which run through the cavernous sinus, the abducens nerve is the most vulnerable to compression by schwannomas of the Gasserian ganglion. The reason is that it takes its course between the intracavernous portion of the internal carotid artery and the Gasserian ganglion in the lower portion of the cavernous sinus, whereas the third and fourth cranial nerves take their courses more superiorly, away from the trigeminal nerve (Fig. 5). The common feature in our two cases was that abducens nerve palsy had brought the patient to the hospital in the early stage of the illness. Trigeminal schwannoma should be included in the list of aetiological factors in the differential diagnosis of unilateral abducens nerve palsy. Occasionally the tumour may grow un- noticed to a considerable size, as in case 6 of Olive and Svien's series (1957), where an asymptomatic schwannoma $25 \times 25 \times 20 \mathrm{~mm}$ was found in the Gasserian ganglion at necropsy of a patient who died of Hodgkin's disease.

Radiological investigations are extremely important in the early diagnosis of trigeminal schwannomas (Palacios and MacGee, 1972; Miyagami et al., 1974; Levinthal and Bentson, 1976). The most constant findings on plain skull films are destruction of the petrous apex and enlargement of the foramina ovale and rotundum (Basauri and Asenjo, 1967). However, all these findings are late signs and were not conspicuous in our two cases. Characteristic changes may be demonstrated by angiography. The intracavernous portion of the internal carotid artery is in most cases displayed anteriorly, medially, and inferiorly (Westberg, 1963; Glasauer and Tandon, 1969; 


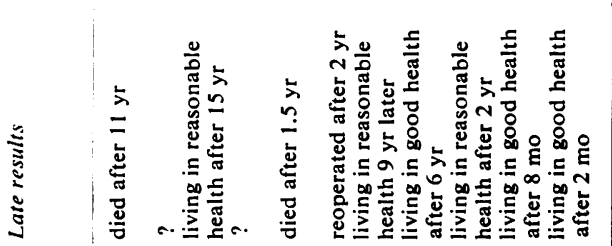

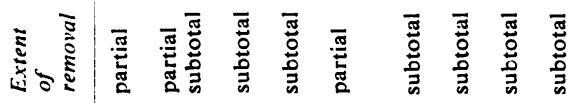

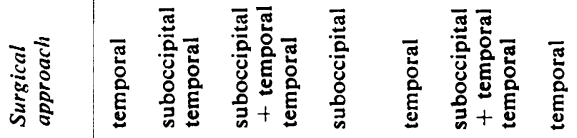

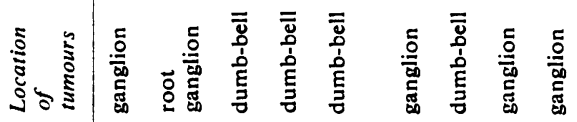

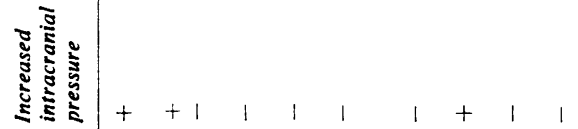

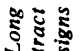

ยั้

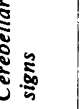

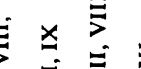

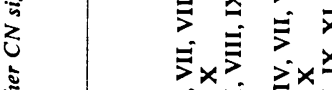

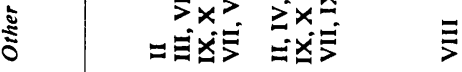

ऽ产 $+++1++1+++$

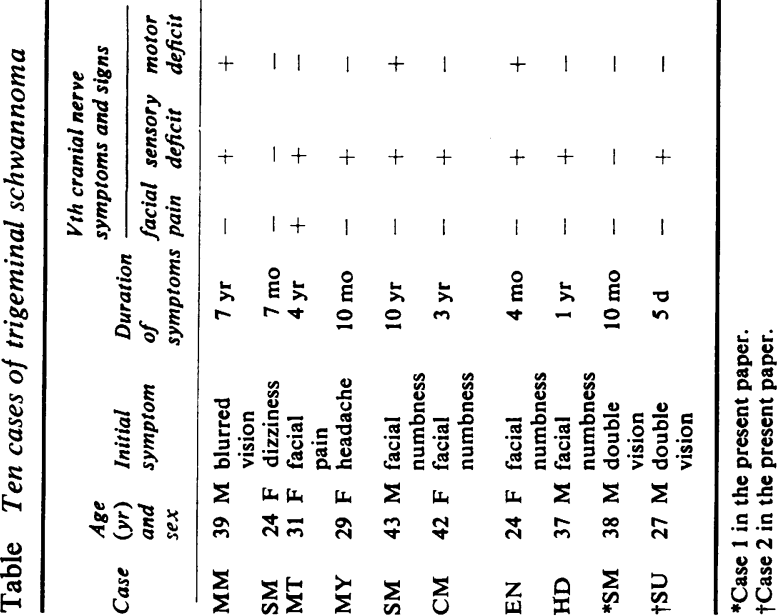

을

$\stackrel{0}{N}$

뭉

ㅇํ을

느

무

$8 \stackrel{0}{\circ}$

흘

흑음

$\vec{V}$

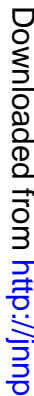

훙.

을

으

음

ज

Nิ 


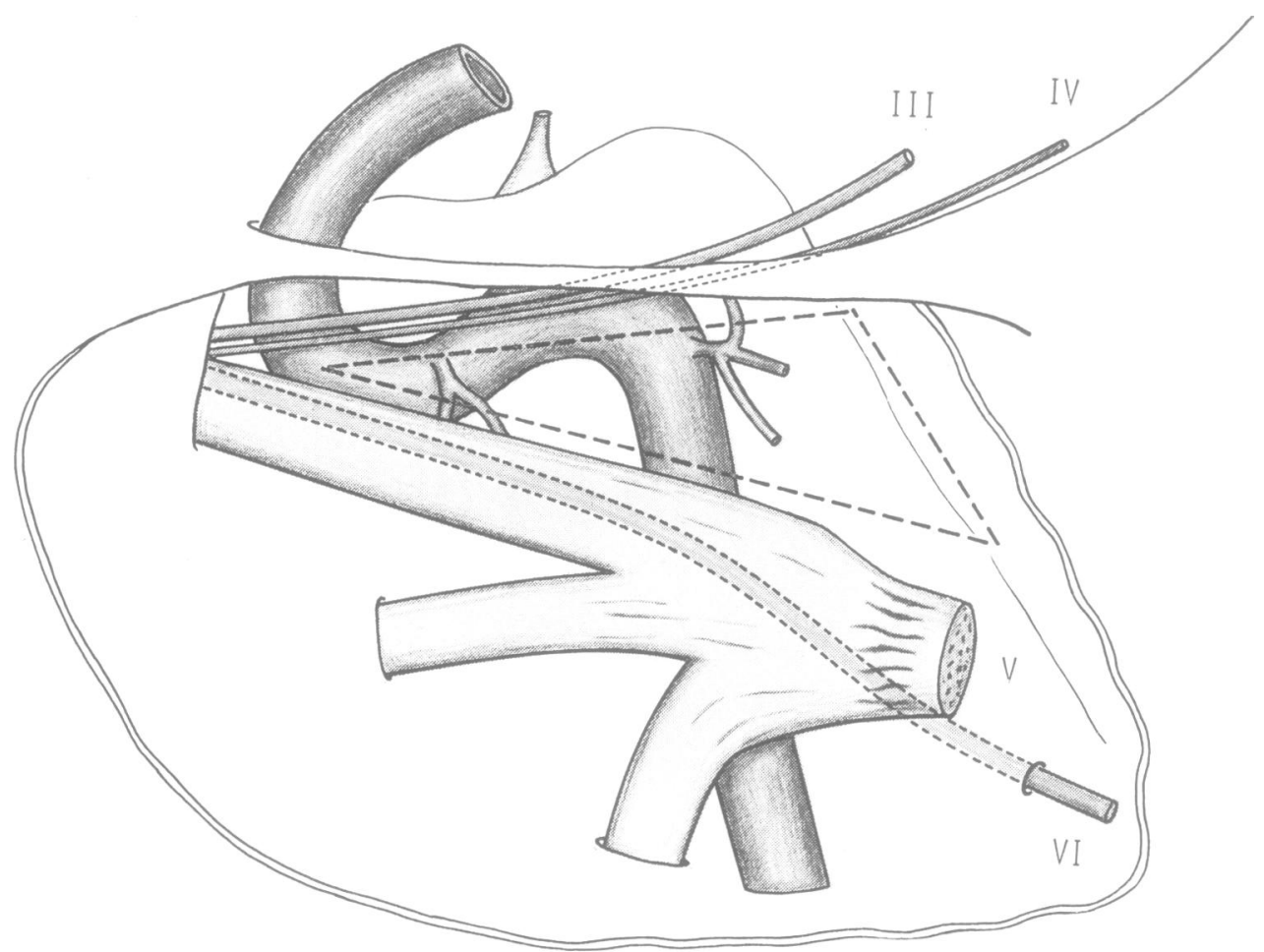

Fig. 5 Diagram sketch of intracavernous portion of internal carotid artery. The third and fourth nerves run above and the fifth and sixth below triangular space outlined in the dotted line. Redrawn from Parkinson (1965).

Wüllenweber et al., 1976). In both of our cases, however, the intracavernous portion was displaced medially, superiorly and posteriorly, indicating that the tumour was growing mainly below and anterior to the internal carotid artery. We believe that the direction of tumour growth is closely related to the early manifestation of abducens nerve palsy. Similar angiographic changes were described in case 3 of Westberg's series (1963) but without clinical details. Angiography was not performed in case 3 of Schisano and Olivecrona's series (1960). It was performed but not fully described in case 9 of Schisano and Olivecrona's series (1960). Angiography in Gordy's case (1965) revealed straightening and elevation of the carotid syphon with an irregular, feathery vascular stain in the medial temporal region. A similar tumour stain was seen in both of our cases. These pathological vessels may represent actual tumour vessels or enlargement of the branches of the artery of the inferior cavernous sinus. Retrograde jugular venography may provide important information on cavernous involvement with the tumour.
Pneumoencephalography revealed a slight elevation of the temporal horn in case 1 reported above. This procedure, however, will certainly be replaced to some extent by computerised tomographic scanning in the future.

Schwannomas in the middle cranial fossa can be excised through a temporal craniotomy. We prefer an intradural approach to obtain a better exposure. Total removal of the tumour is the treatment of choice, although great care is necessary to avoid damage to the cranial nerves and the internal carotid artery in the cavernous sinus. We had to content ourselves with incomplete removal in both cases. Fortunately, however, the long-term result of partial removal is reported to be rather good (Schisano and Olivecrona, 1960; Northfield, 1973).

\section{References}

Arseni, C., Dumitrescu, L., and Constantinescu, A. (1975). Neurinomas of the trigeminal nerve. Surgical Neurology, 4, 497-503. 
Basauri, L., and Asenjo, A. (1967). Trigeminal neurinomas. Acta Neurochirurgica, 17, 1-6.

Glasauer, F. E., and Tandon, P. N. (1969). Trigeminal neurinomas in adolescents. Journal of Neurology, Neurosurgery, and Psychiatry, 32, 562-568.

Gordy, P. D. (1965). Neurinoma of the Gasserian ganglion. Report of a case and review of the literature. Journal of Neurosurgery, 22, 90-94.

Jefferson, G. (1955). The trigeminal neurinomas with some remarks on malignant invasion of the Gasserian ganglion. Clinical Neurosurgery, 1, 11-54.

Knudsen, V., and Kolze, V. (1972). Neurinoma of the Gasserian ganglion and the trigeminal root. Acta Neurochirurgica, 26, 159-164.

Krayenbühl, H. (1936). Primary tumours of the root of the fifth cranial nerve: their distinction from tumours of the Gasserian ganglion. Brain, 59, 337352.

Levinthal, R., and Bentson, J. R. (1976). Detection of small trigeminal neurinomas. Journal of Neurosurgery, 45, 568-575.

Miyagami, M., Goto, T., Sugawara, T., Tsubokawa, T., and Moriyasu, N. (1974). Trigeminal neurinoma: ganglion type and root type. Neurological Surgery, 2, 63-70). (In Japanese.)

Northfield, D. W. C. (1973). The Surgery of the Central Nervous System. Blackwell Scientific Publications: Oxford.

Olive, I., and Svien. H. J. (1957). Neurofibromas of the fifth cranial nerve. Journal of Neurosurgery, 14, 484-505.

Palacios, E., and MacGee, E. E. (1972). The radiographic diagnosis of trigeminal neurinomas. Journal of Neurosurgery, 36, 153-156.

Parkinson, D. (1965). A surgical approach to the cavernous portion of the carotid artery: anatomical studies and case report. Journal of Neurosurgery, 23, 474-483.

Schisano, G., and Olivecrona, H. (1960). Neurinomas of the Gasserian ganglion and trigeminal root. Journal of Neurosurgery, 17, 306-322.

Westberg, G. (1963). Angiographic changes in neurinoma of the trigeminal nerve. Acta Radiologica Diagnostica, 1, 513-520.

Wüllenweber, R., Traupe, H., and Schwarz, G. (1976). Angiographic demonstration of a trigeminal neurinoma. Neuroradiology, 10, 225-229. 